\title{
Ovarian reserve markers in unexplained infertility patients treated with clomiphene citrate during intrauterine insemination
}

\author{
Gokalp Oner ${ }^{1}$, Pasa Ulug ${ }^{1}$, Ferhan Elmali²
}

${ }^{1}$ Department of Obstetrics and Gynecology, Erzincan University, Erzincan, Turkey ${ }^{2}$ Department of Statistics, Erciyes University, Kayseri, Turkey

Submitted: 31 October 2013

Accepted: 12 January 2014

Arch Med Sci 2015; 11, 6: 1250-1254

DOI: 10.5114/aoms.2014.41167

Copyright $\odot 2015$ Termedia \& Banach

\author{
Corresponding author: \\ Gokalp Oner MD \\ Department of Obstetrics \\ and Gynecology \\ Erzincan University \\ Erzincan, Turkey \\ Phone: +9005062346822 \\ E-mail: onerg@yahoo.com
}

\section{Abstract}

Introduction: The aim of this retrospective case control study was to identify predictors of ovarian response and pregnancy outcomes in intrauterine insemination (IUI).

Material and methods: One hundred women undergoing IUI cycles with clomiphene citrate were enrolled. The number of antral follicles and the total ovarian volume by ultrasound, and the basal levels of follicle-stimulating hormone (FSH), estradiol, and inhibin B on cycle day 3 were measured in groups that were divided according to ovarian response. The tests were also evaluated according to ovarian response and pregnancy outcomes. All analyses were performed using the Statistical Package for the Social Sciences, version 15.0 (SPSS, Chicago, IL, USA).

Results: The antral follicle count (AFC) was the best single predictor for ovarian response and pregnancy outcomes. The sensitivity and specificity for prediction of ovarian response were $81 \%$ and $78 \%$ for AFC at an optimum cutoff value of $\leq 13.1$. Age was negatively correlated with ovarian volume $(r=-0.280, p=0.021)$ and AFC $(r=-0.358, p=0.003)$. Increasing FSH was associated with a reduction in AFC $(r=-0.273, p=0.025)$. The AFC was significantly correlated with ovarian volume $(r=0.660, p<0.0001)$ and FSH $(r=-0.273, p=0.03)$.

Conclusions: Our data demonstrate that the AFC provides better prognostic information on the occurrence of ovarian response during clomiphene citrate stimulation for IUI.

Key words: antral follicle count, ovarian reserve, intrauterine insemination, clomiphene citrate, unexplained infertility.

\section{Introduction}

Infertility affects approximately $15-20 \%$ of reproductive aged couples [1]. Primary infertility is a term used to describe a couple that has never been able to conceive a pregnancy after a minimum of 1 year of attempting to do so through unprotected intercourse [2]. Causes of infertility include a wide range of physical as well as emotional factors [3]. Intrauterine insemination (IUI) with ovarian stimulation has been widely used as the primary therapeutic modality for infertility, especially that which is unexplained or caused by nonsevere male factor, anovulation or cervical mucus hostility. Moreover, IUI with controlled ovarian hyperstimulation is 
commonly used as first-line treatment for couples with unexplained subfertility [4].

Ovarian reserve is the major factor in human fertility potential. Age is considered to be the single most important factor in determining quality and quantity of ovarian reserve. The most common tests are basal tests for follicle-stimulating hormone (FSH), luteinizing hormone ( $\mathrm{LH})$, estradiol (E2) and inhibin B, or dynamic endocrine tests such as the clomiphene citrate challenge test and gonadotrophin analogue stimulating test [5]. In recent years, great attention has been devoted to direct tests such as the antral follicle count (AFC), ovarian volume and ovarian biopsy results. The basal FSH concentration is the most common test utilized for ovarian screening. More recently, anti-Mullerian hormone (AMH) has been used by various groups to assess the ovarian reserve [6]. However, the availability of multiple ovarian reserve markers suggests that none is ideal [7].

Consequently, ovarian reserve can be screened using various tests. The aim of the present study is to compare the value of different tests for measuring ovarian reserve in unexplained infertility. In this study, we also evaluated the commonly used tests predicting ovarian reserve and IUI outcomes in couples with unexplained infertility.

\section{Material and methods}

In this study, a total of 127 IUI cycles in 70 couples (group 1) with unexplained infertility were evaluated. Data were collected from the records of 100 couples with unexplained infertility that underwent IUI in the Gynecology and Obstetric Medicine Unit of Erzincan University in 2013. Thirty women (group 2) had no ovarian response with ovarian stimulation.

Unexplained infertility was defined as infertility for couples with no definite reason. Patients with at least one patent tube in hysterosalpingography or laparoscopy and husbands with normal sperm parameters according to the World Health Organization classification were included. Women with myomas, endometriosis and uterine anomalies were excluded from the study. Patients with a basal FSH level $>12 \mathrm{mIU} / \mathrm{ml}$, a maternal age $>40$ years, polycystic ovarian syndrome or a coexisting chronic disease such as hyper- or hypothyroidism, diabetes mellitus or any history of previous reproductive surgery were also excluded from the study. The inclusion criterion was unexplained infertility couples without any systemic or anatomic disease.

They underwent venipuncture at approximately 08:00 hours for baseline pretreatment antral follicle count assessment in the early follicular phase (days 2-4) of the menstrual cycle. Serum samples were stored at $-20^{\circ} \mathrm{C}$ and assayed for $\mathrm{LH}, \mathrm{FSH}, \mathrm{E} 2$, and inhibin B. In the same morning of the blood tests, the total numbers of antral follicles measuring 2$10 \mathrm{~mm}$ in diameter were counted by the same operator. A 7.5 MHz transvaginal probe was used in all examinations. Ovarian volume was calculated with the equation of an ellipsoid ( $0.526 \times$ length $\times$ height $\times$ width). The inclusion criteria were age between 20-39 years, both ovaries present on transvaginal ultrasound scan, no previous ovarian operation, adequate visualization of ovaries on transvaginal ultrasound, no exposure to cytotoxic drugs or pelvic radiation therapy, and no current hormone therapy.

For controlled ovarian hyperstimulation, $50 \mathrm{mg}$ of clomiphene citrate (CC) was given orally from day 5 to day 10. Patients were monitored by transvaginal folliculometry. When one follicle reached at least $\geq 16 \mathrm{~mm}$ in diameter, a $250 \mu \mathrm{g}$ of recombinant human chorionic gonadotropin (hCG; Ovitrelle, Serono) was subcutaneously administered. IUI with the husband's semen was performed $36 \mathrm{~h}$ after the administration of hCG. After semen preparation, a total of $1 \mathrm{ml}$ of sperm preparation was slowly injected into the uterine cavity. Clinical pregnancy was defined as the ultrasound observation of fetal heart movements at 7-8 weeks of gestation. Ovarian response positivity was determined when at least one follicle reached $\geq 16 \mathrm{~mm}$ in diameter after ovarian stimulation by CC. If there was no follicle $\geq 16 \mathrm{~mm}$ in diameter, it was determined as ovarian response negativity.

\section{Statistical analysis}

Numerical variables were presented as means \pm SD. After the confirmation of normal distribution, the $t$ test or Mann-Whitney $U$ test was used to compare the values of the groups. Spearman correlation coefficients were used to assess the relationships between the parameters. Logistic regression analysis was used to evaluate the value for the prediction of oocytes $\geq 16 \mathrm{~mm}$ in diameter. Value of $p \leq 0.05$ was considered statistically significant. All analyses were performed using the Statistical Package for the Social Sciences, version 15.0 (SPSS, Chicago, IL, USA).

\section{Results}

One hundred women were divided into two groups according to their response to ovarian stimulation. Seventy women had oocytes $\geq 16 \mathrm{~mm}$ in diameter $(70 \%)$, while 30 women had no ovarian response (30\%). Additionally, group 1 was analyzed for the pregnancy outcomes.

Nine $(12.8 \%)$ of 70 patients were pregnant. The chance of pregnancy increased below the age of 35 (93\% sensitivity, $33.3 \%$ specificity), below 7 of FSH level (73\% sensitivity, $55.6 \%$ specificity), above 4.23 of ovarian volume $(91.5 \%$ sensitivity, $55.6 \%$ specificity), and above 12 of AFC ( $85 \%$ 
sensitivity, $77.8 \%$ specificity) according to receiver operating characteristic (ROC) analysis. Moreover, there was no statistically significant difference in age, FSH, AFC or ovarian volume between the groups $(p<0.05)$.

Table I summarizes patients' demographics, basal FSH, AMH, and AFC in the two study groups. There was no difference between the ages of the groups. Body mass index (BMI) was similar in both groups. Group 2 had a lower total AFC $(p<0.001)$ and smaller ovaries $(p<0.05)$, but there was no statistically significant difference between the serum

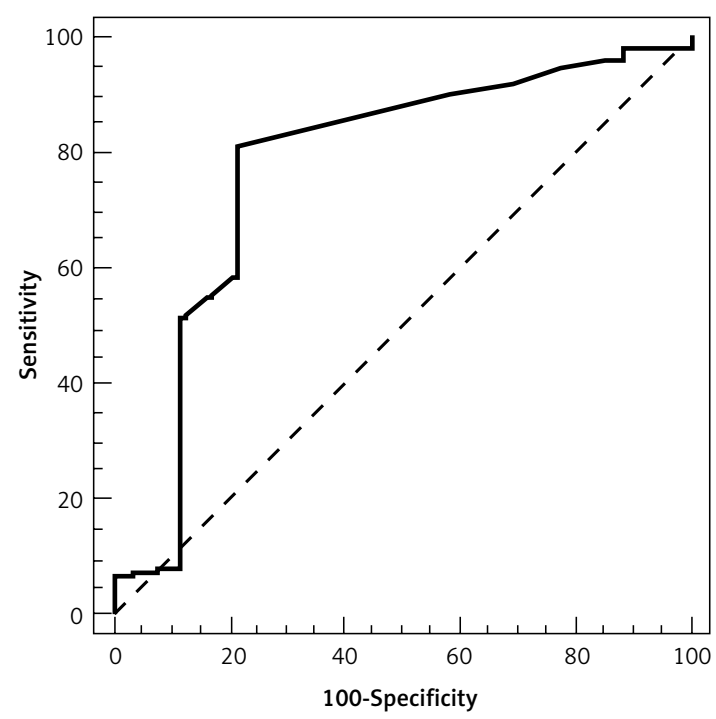

Figure 1. ROC curve analysis of antral follicle count
FSH levels of groups. The other endocrine (basal $\mathrm{LH}, \mathrm{E2}$, and inhibin-B) markers were similar in both groups. Multivariate logistic regression analysis of the variables was performed for the prediction of oocytes $\geq 16 \mathrm{~mm}$ in diameter. The AFC was the only significant predictor on multivariate analysis (1.121, 95\% Cl: $1.014-1.372 ; p=0.035)$. In group 1 , similar results were obtained for prediction of pregnancy, with several of the measures being predictive on univariate logistic regression analyses, which again revealed AFC as a significant predictor (Table II). Additionally, ovarian volume was the other significant predictor of pregnancy (Table II). The ROC curve analysis (Figure 1) showed that AFC predicted the ovarian response, as demonstrated by a similar area under the curve (AUC) $(p<0.001)$. The optimum cutoff level of AFC was 13.1 with $81.4 \%$ sensitivity (95\% Cl: 69.1-90.3), $77.8 \%$ specificity (95\% Cl: 40.1-96.5), 3.66 positive likelihood ratio, and 0.24 negative likelihood ratio.

Table III summarizes the correlation of the clinical and hormonal parameters of the 70 patients. Age was negatively correlated with ovarian volume $(r=-0.280, p=0.021)$, and AFC $(r=-0.358$, $p=0.003)$. Increasing FSH was associated with a reduction in AFC $(r=-0.273, p=0.025)$. The AFC was significantly correlated with ovarian volume $(r=0.660, p<0.0001)$, and FSH $(r=-0.273, p=0.03)$.

\section{Discussion}

The proportion of couples suffering from unexplained infertility is popularly quoted as $16 \%$, but

Table I. Comparison of baseline clinical, endocrine, and ultrasound characteristics between groups

\begin{tabular}{|c|c|c|c|}
\hline Parameters & Group $1(n=70)$ & Group $2(n=30)$ & Value of $p$ \\
\hline Age [years] & $30.4 \pm 5.2$ & $31.5 \pm 4.6$ & 0.27 \\
\hline Body mass index $\left[\mathrm{kg} / \mathrm{m}^{2}\right]$ & $24.6 \pm 4.2$ & $24.3 \pm 4.2$ & 0.79 \\
\hline Basal FSH level [IU/I] & $6.9 \pm 2.2$ & $7.4 \pm 3.2$ & 0.28 \\
\hline Basal LH level [IU/I] & $6.3 \pm 3.1$ & $6.9 \pm 3.6$ & 0.16 \\
\hline Basal $E_{2}$ level [pmol/l] & $66.6 \pm 358.0$ & $67.9 \pm 117.7$ & 0.31 \\
\hline Inhibin-B [pg/ml] & $52.9 \pm 38.1$ & $68.5 \pm 107.6$ & 0.94 \\
\hline Total antral follicle count & $18.7 \pm 6.4$ & $12.2 \pm 8.7$ & 0.009 \\
\hline Mean ovarian volume $\left[\mathrm{cm}^{3}\right]$ & $10.1 \pm 4.2$ & $7.9 \pm 3.5$ & 0.04 \\
\hline
\end{tabular}

Values are presented as mean $\pm S D$

Table II. Univariate analysis of baseline markers of ovarian reserve for prediction of pregnancy outcomes in group 1

\begin{tabular}{|lccc|}
\hline Parameters & Odds ratio & $95 \% \mathrm{Cl}$ & Value of $p$ \\
\hline Age & 0.91 & $0.872-1.075$ & 0.33 \\
\hline Basal FSH & 0.707 & $0.627-1.076$ & 0.13 \\
\hline Inhibin-B & 0.997 & $0.981-1.004$ & 0.19 \\
\hline Mean ovarian volume & 1.316 & $1.015-1.707$ & 0.03 \\
\hline Antral follicle count & 1.325 & $1.055-1.536$ & 0.01 \\
\hline
\end{tabular}


Table III. Spearman correlation coefficient of clinical and hormonal parameters

\begin{tabular}{|lcccc|}
\hline Variable & Age & FSH & OV & AFC \\
\hline Age & - & 0.202 & -0.280 & -0.358 \\
\hline FSH & 0.102 & - & -0.189 & -0.273 \\
\hline OV & 0.021 & 0.125 & - & 0.660 \\
\hline AFC & 0.003 & 0.025 & $<0.0001$ & - \\
\hline
\end{tabular}

the range varies from $0 \%$ to $37 \%$ [8]. Clomiphene citrate is the first-line treatment for unexplained infertility [9]. Although the effectiveness of the treatment was evaluated, a recently published review showed that clomiphene citrate may improve fertility as much as 2-fold [8]. In our study the pregnancy rate was $12.8 \%$. The pregnancy rate in IUI cycles has varied between $11.4 \%$ and $12.6 \%$ [10]. In this study, we examined the relation between the prediction of ovarian reserve tests and the pregnancy outcomes and mature follicle in unexplained infertility patients treated by CC with IUI.

An ideal ovarian reserve parameter should be easily measurable, minimally invasive, and inexpensive and should have good predictive values [11]. In infertility treatment with ovulation induction and IUI, the fertility expert has limited parameters to predict the likelihood of success. Our study confirmed the efficacy of AFC as a biomarker of ovarian function. We observed an inverse correlation between AFC and serum FSH levels measured at the early follicular phase. We also noted that high levels of AFC were positively correlated with pregnancy after IUI in unexplained infertility. To our knowledge, this is the first study to evaluate the prediction of mature follicle and pregnancy with ovarian reserve markers in intrauterine insemination for treatment of unexplained infertility with CC.

Age is considered to be the single most important factor in determining ovarian reserve [12]. In our study, increased age ( $\geq 35$ years) negatively affected the pregnancy outcomes ( $93 \%$ sensitivity, $33.3 \%$ specificity); however, there was no statistically significant effect to predict the pregnancy.

The FSH is the most studied and used endocrine test in determining ovarian reserve [5]. Previous reports have shown that elevated 3 FSH levels on the basal day were related to lower pregnancy rates [13]. However, this criterion cannot indicate a poor ovarian response with normal basal FSH levels. Van der Steeg et al. studied the predictive value of basal FSH for spontaneous pregnancy occurrence in ovulatory subfertile women younger than 40 years and observed reduced chances when the levels exceeded $8 \mathrm{IU} / \mathrm{l}$, whereas no association could be determined for lower levels [14]. In a prospective study, Haadsma et al. found that basal FSH and AFC were significant predictors of spontaneous pregnancy [15]. Our study showed that FSH may not predict ovarian response or pregnancy in unexplained infertility couples treated with IUI, but pregnancy rates decreased when FSH levels exceeded 7 IU/l. Increasing FSH was also associated with a reduction in AFC.

Inhibin $B$ has been postulated as an alternative endocrine marker of ovarian reserve. It has been suggested that measuring inhibin B concentrations would provide a more direct assessment of ovarian reserve [16]. However, other studies did not recommend the use of inhibin $B$ alone as a reliable predictor of ovarian reserve $[17,18]$. Our data showed that inhibin B did not predict the response to ovulation induction and pregnancy in IUI cycles of unexplained infertility stimulated with CC.

Ovarian volume and AFC values can be useful markers to determine ovarian reserve [19]. Erdem et al. suggest that transvaginal ultrasonography rather than hormonal parameters is the preferred method for ovarian reserve determination, as ultrasound assessment of ovarian volume and the AFC confer a stronger correlation with chronological aging than day $3 \mathrm{FSH}$ level indices and aging [20]. Additionally, several investigators have reported the effectiveness of AFC and ovarian volume in predicting ovarian response to hormonal stimulation in in vitro fertilization (IVF) [21, 22]. In another study, AFC demonstrated the best correlation with women's age [23]. Women undergoing their first IVF cycle were evaluated with a battery of tests to compare several basal ovarian reserve markers [24]. Measurements were performed to determine the number of antral follicles, total ovarian volume, basal FSH, E2, and inhibin B on cycle day 3. The AFC was the best single predictor for a poor ovarian response [24]. However, the performance of the AFC for predicting failure to achieve pregnancy is poor [23-25]. Our results revealed that AFC displayed a significant positive correlation between ovarian volume and a negative correlation between age and FSH. Additionally, AFC was the most predictive test for the ovarian response and pregnancy outcomes. This study suggests that the AFC provides an optimum sensitivity and specificity of 0.81 and 0.78 , respectively, at a cutoff value of 13.1 or less for the prediction of ovarian response. Ovarian volume was the other significant predictor of pregnancy but not ovarian response. 
The IUI is the first step and is important for infertile women before IVF cycles [26]. The prediction of the treatment outcomes is pivotal for the treatment procedures in infertile therapy. Although there have been many trials for the prediction of IVF outcomes and the relation between ovarian reserve and ovarian response, there is no study to predict ovarian response in IUI cycles. Our study evaluated the tests of ovarian reserve markers in IUI treatment with CC and illustrated that AFC was more powerful to show an ovarian response. This study might help to estimate the tests of ovarian reserve before IUI cycles.

In conclusion, pretreatment AFC was the most significant predictor of ovarian response and pregnancy outcomes for ovarian stimulation with CC during IUI. Additionally, ovarian volume might predict the pregnancy outcomes. Our study is the first to demonstrate this in unexplained infertility couples. More prospective studies are needed in order to confirm the predictive value of the markers.

\section{Conflict of interest}

The authors declare no conflict of interest.

\section{References}

1. Roudebush WE, Kivens WJ, Mattke JM. Biomarkers of ovarian reserve. Biomark Insights 2008; 3: 259-68.

2. Evers JL. Female subfertility. Lancet 2002; 360: 151-9.

3. Rouchou B. Consequences of infertility in developing countries. Perspect Public Health 2013; 133: 174-9.

4. Steures P, van der Steeg JW, Hompes PG, et al.; Collaborative Effort on the Clinical Evaluation in Reproductive Medicine. Intrauterine insemination with controlled ovarian hyperstimulation versus expectant management for couples with unexplained subfertility and an intermediate prognosis: a randomised clinical trial. Lancet 2006; 368: 216-21.

5. Broekmans FJ, Kwee J, Hendriks DJ, Mol BW, Lambalk CB. A systematic review of tests predicting ovarian reserve and IVF outcome. Hum Reprod Update 2006; 12: 685-718.

6. Broer SL, Dólleman M, Opmeer BC, Fauser BC, Mol BW, Broekmans FJ. AMH and AFC as predictors of excessive response in controlled ovarian hyperstimulation: a meta-analysis. Hum Reprod Update 2011; 17: 46-54.

7. Jirge PR. Ovarian reserve tests. J Hum Reprod Sci 2011; 4: 108-13.

8. Ray A, Shah A, Gudi A, Homburg R. Unexplained infertility: an update and review of practice. Reprod Biomed Online 2012; 24: 591-602.

9. Pandian Z, Gibreel A, Bhattacharya S. In vitro fertilisation for unexplained subfertility. Cochrane Database Syst Rev 2012; 4: CD003357.

10. ESHRE Capri Workshop Group. Intrauterine insemination. Hum Reprod Update 2009; 15: 265-77.

11. Kwee J, Schats R, McDonnell J, Schoemaker J, Lambalk CB. The clomiphene citrate challenge test versus the exogenous follicle-stimulating hormone ovarian reserve test as a single test for identification of low responders and hyperresponders to in vitro fertilization. Fertil Steril 2006; 85: 1714-22.
12. Sahakyan M, Harlow BL, Hornstein MD. Influence of age, diagnosis, and cycle number on pregnancy rates with gonadotropin-induced controlled ovarian hyperstimulation and intrauterine insemination. Fertil Steril 1999; 72: 500-4.

13. Martin JS, Nisker JA, Tummon IS, Daniel SA, Auckland JL, Feyles V. Future in vitro fertilization pregnancy potential of women with variably elevated day 3 follicle-stimulating hormone levels. Fertil Steril 1996; 65: 1238-40.

14. van der Steeg JW, Steures P, Eijkemans MJ, et al. Predictive value and clinical impact of basal follicle-stimulating hormone in subfertile, ovulatory women. J Clin Endocrinol Metab 2007; 92: 2163-8.

15. Haadsma ML, Groen H, Fidler V, et al. The predictive value of ovarian reserve tests for spontaneous pregnancy in subfertile ovulatory women. Hum Reprod 2008; 23: 1800-7.

16. Creus $M$, Peñarrubia J, Fábregues $F$, et al. Day 3 serum inhibin B and FSH and age as predictors of assisted reproduction treatment outcome. Hum Reprod 2000; 15: 2341-6.

17. Corson SL, Gutmann J, Batzer FR, Wallace H, Klein N, Soules MR. Inhibin-B as a test of ovarian reserve for infertile women. Hum Reprod 1999; 14: 2818-21.

18. Fanchin R, Schonäuer LM, Righini C, Guibourdenche J, Frydman R, Taieb J. Serum anti-Müllerian hormone is more strongly related to ovarian follicular status than serum inhibin B, estradiol, FSH and LH on day 3. Hum Reprod 2003; 18: 323-7.

19. Flaws JA, Langenberg P, Babus JK, Hirshfield AN, Sharara Fl. Ovarian volume and antral follicle counts as indicators of menopausal status. Menopause 2001; 8: 175-80.

20. Erdem M, Erdem A, Biberoglu K, Arslan M. Age-related changes in ovarian volume, antral follicle counts and basal follicle stimulating hormone levels: comparison between fertile and infertile women. Gynecol Endocrinol 2003; 17: 199-205.

21. Bancsi LF, Broekmans FJ, Eijkemans MJ, de Jong FH, Habbema JD, te Velde ER. Predictors of poor ovarian response in in vitro fertilization: a prospective study comparing basal markers of ovarian reserve. Fertil Steril 2002; 77: 328-36.

22. Chang MY, Chiang CH, Hsieh TT, Soong YK, Hsu KH. Use of the antral follicle count to predict the outcome of assisted reproductive technologies. Fertil Steril 1998; 69: 505-10.

23. Ng EH, Yeung WS, Fong DY, Ho PC. Effects of age on hormonal and ultrasound markers of ovarian reserve in Chinese women with proven fertility. Hum Reprod 2003; 18: 2169-74.

24. Bancsi LF, Broekmans FJ, Eijkemans MJ, de Jong FH, Habbema JD, te Velde ER. Predictors of poor ovarian response in in vitro fertilization: a prospective study comparing basal markers of ovarian reserve. Fertil Steril 2002; 77: 328-36.

25. Hendriks DJ, Mol BW, Bancsi LF, Te Velde ER, Broekmans FJ. Antral follicle count in the prediction of poor ovarian response and pregnancy after in vitro fertilization: a meta-analysis and comparison with basal follicle-stimulating hormone level. Fertil Steril 2005; 83: 291-301.

26. Mao GH, Feng Z,He Y, Huang Y. Comparisons of the effects of long-acting and short-acting GnRH agonists on embryo quality, endometrial thickness and pregnancy rate in human in vitro fertilization. Arch Med Sci 2014; 10: 161-6. 